\title{
http://dx.doi.org/10.5007/2175-7984.2012v11n20p49 A gordura trans: entre as
controvérsias científicas e as
estratégias da indústria alimentar
}

Marília Luz David' Julia S. Guivant²

\section{Resumo}

Entre os riscos alimentares modernos, o decorrente da gordura trans destaca-se como um dos que se tornaram parte do cotidiano. A gordura trans fabricada industrialmente foi considerada uma alternativa saudável para a produção de alimentos e passou a ser amplamente utilizada pela indústria de alimentos a partir da segunda metade do século XX. Esta gordura foi considerada um substituto mais saudável para a gordura animal. No entanto, em 1990 teve início uma controvérsia científica, com a publicação de um artigo em um dos principais periódicos científicos da área médica que relacionou o consumo de gordura trans à ocorrência de doenças cardiovasculares. Neste artigo analisamos primeiro como se consolidou a gordura trans como risco alimentar e quais foram as modificações realizadas pela indústria alimentar. Finalmente apontamos o paradoxo sobre como, apesar da onipresença de rótulos "Livre de gordura trans", esta continua presente no cotidiano, consumida em pequenas quantidades em diferentes produtos.

Palavras-chave: riscos alimentares, rotulagem, regulação de riscos, alimentos saudáveis.

1 Doutoranda do Programa de Pós-graduação em Sociologia Política da Universidade Federal de Santa Catarina (UFSC), Mestre em Sociologia Política (UFSC), integrante do Instituto de Pesquisa em Risco e Sustentabilidade (IRIS). Contato: mariliadavid@hotmail.com.

2 Professora do Departamento de Sociologia e Ciência Política da Universidade Federal de Santa Catarina (UFSC), coordenadora do Instituto de Pesquisa em Risco e Sustentabilidade (IRIS), pesquisadora do CNPq (S.Net). Contato: juliaguivant@gmail.com. 


\section{Introdução}

$\mathrm{D}$ iversos teóricos e pesquisadores sociais renomados (GIDDENS, 2002; LANG; HEASMAN, 2004; NESTLE, 2007; HERRICK, 2009; BURCH; LAWRENCE, 2010) identificam na alta modernidade a relevância do envolvimento íntimo da indústria alimentar com as diversas formas que toma o que se identifica como "vida saudável". Um dos indicadores desta maior atenção dada pela indústria alimentar é que a partir de 2005 o índice Dow Jones de sustentabilidade passou a utilizar os gastos no setor de pesquisa e desenvolvimento na área de saúde e nutrição para acessar o nível de sustentabilidade das empresas (PATTON, 2005). Em 2011 a PepsiCo ocupou o primeiro lugar no ranking desse índice. As iniciativas empresariais com foco na saúde compreendem, por exemplo, sites na internet com informações sobre alimentação e saúde, financiamento de programas esportivos, utilização de alegações de saúde, selos e certificações para alimentos que seguem recomendações nutricionais de órgãos nacionais e internacionais de saúde. Os alimentos com promessas de saúde surgem como uma das principais formas das empresas distinguirem seus produtos para se posicionar no mercado como uma indústria de "bem-estar" (BURCH; LAWRENCE, 2010; CALLON; MÉADEL; RABEHARIOSA, 2002).

Os alimentos sem gordura trans estão entre os produtos que apareceram na última década nesta tendência de alimentos saudáveis, mas apresentam uma especificidade dentro do mercado. Eles vieram a substituir outros alimentos considerados muito saudáveis até seu surgimento: os que continham gordura trans. Por ser proveniente de óleos vegetais hidrogenados, com menores quantidades de gordura saturada, acreditava-se que a gordura trans era uma opção mais saudável à gordura animal - que comprovadamente aumenta o LDL (colesterol ruim) no sangue. Para a indústria alimentar era uma incrível opção para deixar saborosos e com maior prazo de validade toda uma gama de produtos (salgadinhos, biscoitos, bolos etc.) A isto se somam os benefícios vistos pelos restaurantes de fast-food. 
Neste artigo analisamos como se deu este processo de mudança da gordura trans de panaceia para vilã, e como foram se posicionando neste processo importantes atores institucionais e econômicos, como os cientistas e as empresas alimentares. Argumentamos que, a diferença dos alimentos light, diet, funcionais, orgânicos, com menos lactose etc., seria que aqueles que apresentam " $0 \%$ gordura trans" estão atualmente de forma onipresente no mercado pela consolidação do risco da gordura trans como resultado de uma controvérsia científica. Os alimentos "0\% gordura trans" também mostram como a indústria alimentar se reinventa frente à consolidação de riscos que ela mesma cria - neste caso, a gordura trans - para lançar novos produtos, apresentados como mais saudáveis que os vendidos anteriormente por ela mesma, e colocando-se como tomadora de iniciativa neste processo de busca da vida saudável.

\section{Riscos e alimentação}

Se, por um lado, o sistema alimentar moderno, apoiado em sistemas peritos, foi capaz de criar grandes ambientes de segurança e estabilidade para a produção, distribuição e consumo de alimentos no mundo, por outro lado, também provocou o aumento das ansiedades alimentares (GUIVANT, 2000, 2001). Três características fundamentais podem ser destacadas na vivência dos riscos (GIDDENS, 1991), que contribuem para que alguns paradoxos se tornem mais visíveis. Uma primeira característica é a valorização do nutriente como princípio-chave para avaliar os alimentos e reordenar o aconselhamento nutricional. O termo nutricionismo (SCRINIS, 2008) se refere à abordagem que tenta entender os alimentos a partir do nutriente e a sua relação com determinadas condições de saúde. A qualidade do alimento é tratada a partir de sua dimensão bioquímica, enquanto um conjunto de nutrientes que correspondem a certas funções para a saúde. O nutriente é considerado a instância fundamental para julgar a relação entre os alimentos e a saúde do corpo. 


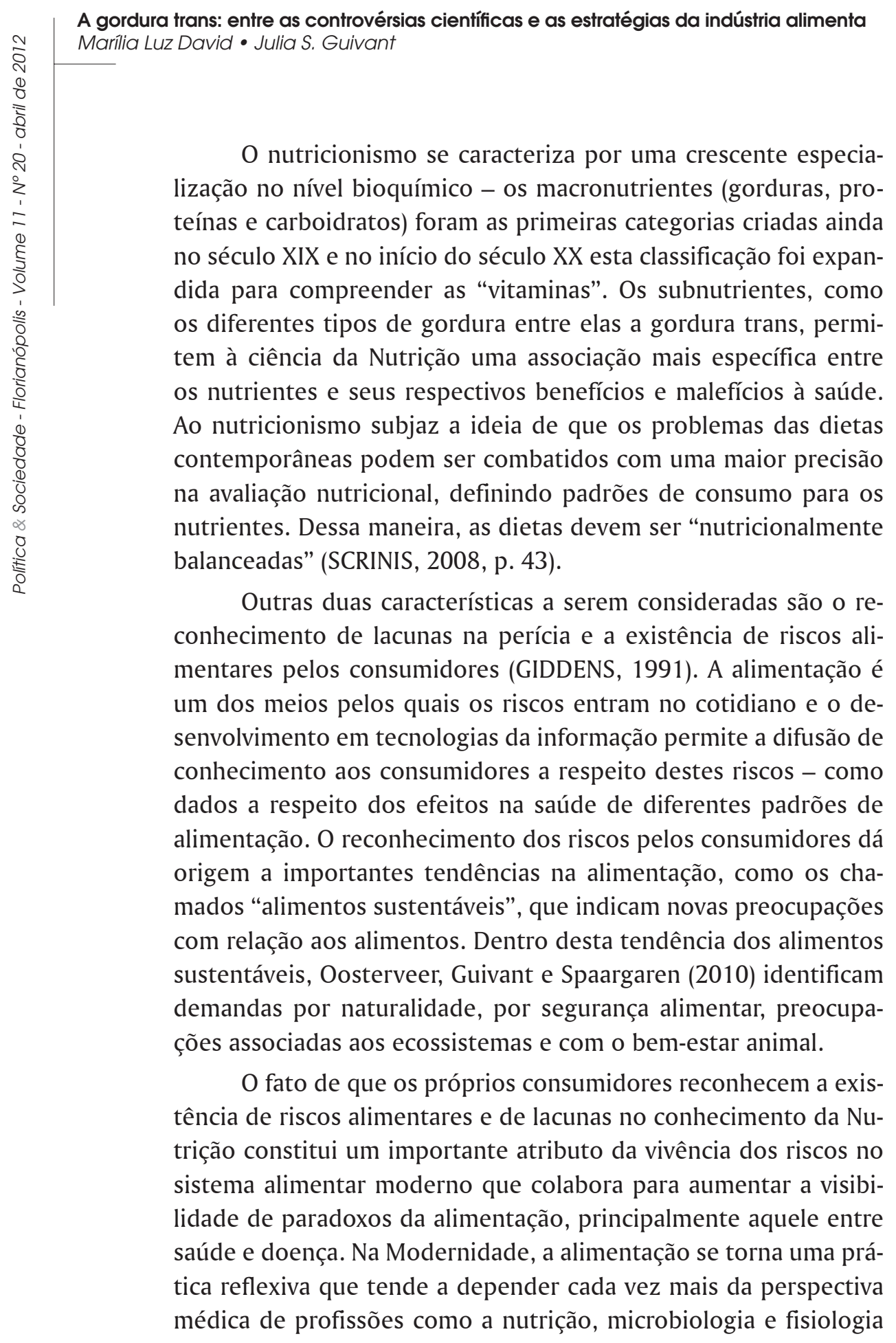


na orientação sobre a relação entre dieta e saúde. Este aconselhamento, por seu turno, passa por uma revisão constante, e o conhecimento em voga de um determinado período pode ser visto retrospectivamente como um engano.

O reconhecimento do risco, da fragilidade e limitação das perícias que operam dentro do sistema alimentar agrava principalmente as ansiedades associadas ao paradoxo saúde-doença, pois estimula a desorientação e as ansiedades em relação ao que comer. Uma pesquisa realizada entre jovens americanas em seis universidades ilustra este quadro: $30 \%$ das participantes responderam que, se fosse possível, ao em vez de comer optariam por ingerir uma pílula que fosse segura, barata e nutricionalmente completa (ROZIN, 1999).

Convivemos com um modelo médico que aproxima o ideal de magreza com um bom estado de saúde enquanto associa a obesidade a uma série de doenças (BEADSWORTH; KEIL, 1997, p. 181). Entretanto, vivemos em circunstâncias contraditórias em que valorizamos o corpo esguio como o ideal de saúde e beleza, ao mesmo tempo em que cresce o peso da população mundial. No Relatório Mundial da Saúde em 2002, a OMS classificou a obesidade como um dos principais riscos contemporâneos à saúde atingindo o nível de epidemia ${ }^{3}$. Ocorre um distanciamento em relação ao que é desejado em termos de saúde e beleza.

A indústria alimentar reconheceu mais recentemente este paradoxo alimentar, assim como é em parte responsabilizada pelo crescimento de doenças crônicas (OMS, 2002a; OMS, 2004). A criação de alimentos com promessas de saúde faz parte de uma estratégia da indústria alimentar para tentar virar o jogo a seu favor.

3 Desde 2002 a OMS privilegia o crescimento das doenças crônicas e a centralidade da alimentação como fator que contribui para o estado de saúde mundial em uma série de publicações. Em 2004 a organização lançou a campanha Global Strategy on Diet, Physical Activity and Health que trouxe recomendações para a saúde pública e destacou a dieta e a falta de exercícios como os principais fatores para o aumento de doenças crônicas. $\mathrm{O}$ aumento das doenças crônicas e a importância da alimentação conferida pela OMS neste período foram dois fatores importantes para conferir um caráter de urgência e relevância à gordura trans como um risco de saúde pública (DAVID, 2011). 
Segundo dados sobre tendências na alimentação do International Food and Information Council, uma instituição de pesquisa financiada pela indústria alimentar, os três principais motivos apresentados por consumidores americanos para tornar sua dieta mais saudável foram o interesse por melhorar seu bem-estar em geral, a saúde física e a perda de peso (INTERNATIONAL FOOD AND INFORMATION COUNCIL, 2008 , p. 8). De maneira semelhante, uma pesquisa realizada no Brasil em 2010, que buscou dados para orientar estratégias de inovação no setor de alimentos e bebidas no país, identificou a tendência "saudabilidade, bem-estar, sustentabilidade e ética" entre as tendências da alimentação no Brasil atualmente (VIALTA et al., 2010).

Com estes traços da vivência dos riscos na alta modernidade e do sistema alimentar moderno discutidos até aqui, procuramos inicialmente caracterizar tendências mais amplas com as quais os alimentos sem gordura trans estão relacionados. A seguir examinamos como esta gordura trans se tornou uma vilã da alimentação por meio de uma controvérsia científica no final do século XX.

\section{A gordura trans}

Até a década de 1950, a maior parte da gordura consumida no mundo era de origem animal, como a banha e a manteiga. Durante a década de 1950 surge o processamento de gorduras a partir de óleos de sementes que permitiu a substituição de gordura animal por óleos vegetais na produção de alimentos e levou a uma das maiores mudanças em padrões de dieta durante o século XX (POPKIN, 2006, p. 561). Para estender a validade dos óleos vegetais e facilitar seu transporte, a indústria alimentar adotou o processo de hidrogenização que torna o óleo vegetal mais consistente.

A maior parte da gordura trans consumida durante o século XX foi criada durante este processo de hidrogenização. A gordura trans é formada por ácidos graxos insaturados. A designação "trans" vem de "transversos" e o nome é referente à ordem da cadeia de átomos do ácido graxo. Na maioria dos ácidos graxos 
encontrados na natureza, por exemplo, os átomos estão distribuídos em posição paralela. No entanto, quando é submetido ao tratamento industrial de hidrogenação, a estrutura química do óleo é modificada, fazendo com que os ácidos graxos fiquem com os átomos em disposição "diagonal" - ou em alinhamento transversal (trans). Ainda que a gordura trans exista em pequenas quantidades em alimentos como o leite e a carne vermelha, a maior parte da gordura trans consumida durante o século XX foi produzida industrialmente por meio do processo de hidrogenação. Os óleos vegetais hidrogenados foram avaliados como mais saudáveis do que a gordura animal por não conterem os mesmos níveis de gordura saturada (POLLAN, 2008).

Durante a década de 1970, o uso de gordura animal na fabricação de alimentos era criticado por peritos da saúde e pela opinião pública nos EUA e em países da Europa por seu alto teor de gordura saturada, associada a efeitos adversos na saúde do coração. No entanto, pouco se conhecia entre os pesquisadores especializados a respeito dos efeitos da gordura trans no organismo, em especial para o coração.

Só nas décadas de 1960 e 1970 surgiram três pesquisas centrais que investigaram os efeitos da gordura trans no colesterol, mas apresentaram conclusões discordantes, o que pode ter diluído seu impacto (ANDERSON; GRANDE; KEYS, 1961; VERGROESEN, 1972; MATTSON; HOLLENBACH; KLIGMAN, 1975). Na década de 1980, a Federação das Sociedades Americanas de Biologia Experimental produziu um relatório comissionado pela Food and Drug Administration (FDA). Este relatório indicou que o consumo de gordura trans não trazia efeitos adversos à saúde (SENTI, 1985) ${ }^{4} \mathrm{e}$ serviu como referência para as políticas do FDA nos anos seguintes (KATAN; KORVER, 2006). Efetivamente, até metade da década de 1990 ainda era possível encontrar produtos fabricados com óleos vegetais hidrogenados e que utilizavam a afirmação " $100 \%$ óleo

4 O FDA se destaca como um dos principais órgãos de regulação de influência internacional para tratar de alimentos. 


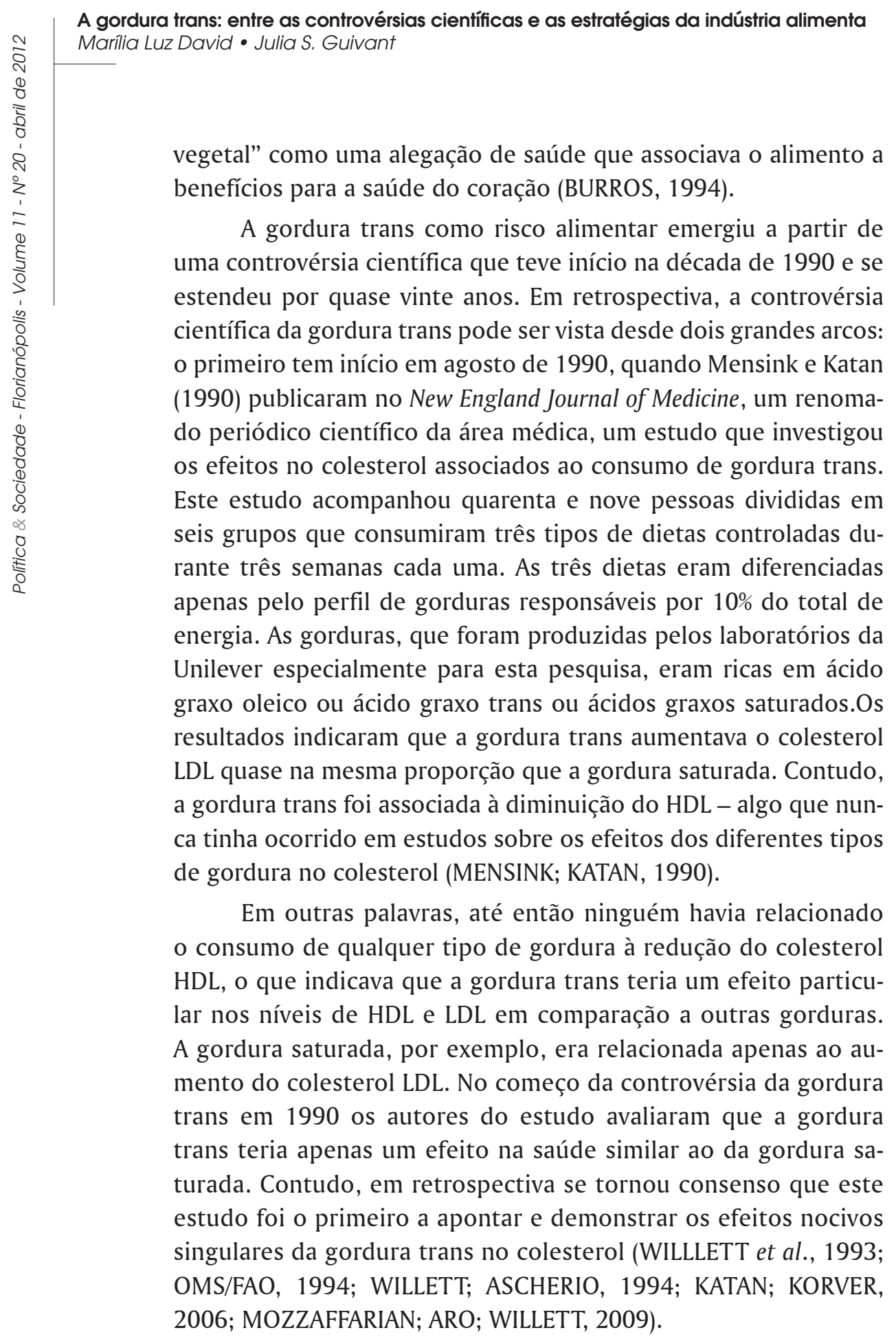


Durante a década de 1990 a discussão sobre os efeitos da gordura trans na saúde humana, especialmente no colesterol, ganhou espaço nos periódicos científicos, mas paulatinamente chamou a atenção de outros atores, como ONGs de consumidores, grandes representantes da indústria alimentar, legisladores, jornalistas. Este primeiro arco se estende até o final da década de 1990 quando o FDA decidiu atualizar a revisão da literatura sobre os efeitos da gordura trans (SCARBROUGH, 1997), e aceitou como evidências estudos que relacionaram a gordura trans à ocorrência de doenças cardiovasculares. As principais pesquisas referenciadas foram a de Mensink e Katan (1990) já citadas anteriormente, o Nurses' Health Study realizado pela Universidade de Harvard desde 1976 e um dos principais estudos epidemiológicos já produzidos (WILLETT et al., 1993; HU et al., 1997), assim como uma pesquisa do Departamento de Agricultura Americano publicada em 1994 (JUDD et al., 1994). O DanishNutritionCouncil em 1995 (STENDER et al., 1995) foi responsável por outro estudo fundamental para o FDA nesta ocasião, em que a autoridade nacional de saúde dinamarquesa recomendou que o consumo de gordura trans fosse reduzido para os menores níveis possíveis. Após esta revisão de literatura realizada pelo FDA, a agência anuncia em 1999 planos que tornariam obrigatória a rotulagem da gordura trans em alimentos (DAVID, 2011).

A introdução das primeiras regras para a rotulagem nutricional da gordura trans no Canadá e nos Estados Unidos a partir de 2003 e 2006 respectivamente, assim como a decisão do governo dinamarquês de banir alimentos com gordura trans em 2003 foram importantes marcos em legislações nacionais para a consolidação da gordura trans como risco (DAVID, 2011). Estes foram momentos que aos poucos fecharam parte da controvérsia científica (LATOUR, 2000), pois a associação positiva entre o consumo de gordura trans e os prejuízos à saúde subjaze à formulação de tais medidas.

A revisão do aconselhamento nutricional da OMS a partir de 2003, que passou a classificar a gordura trans como um dos fatores que contribuem para a ocorrência de doenças cardiovasculares e 


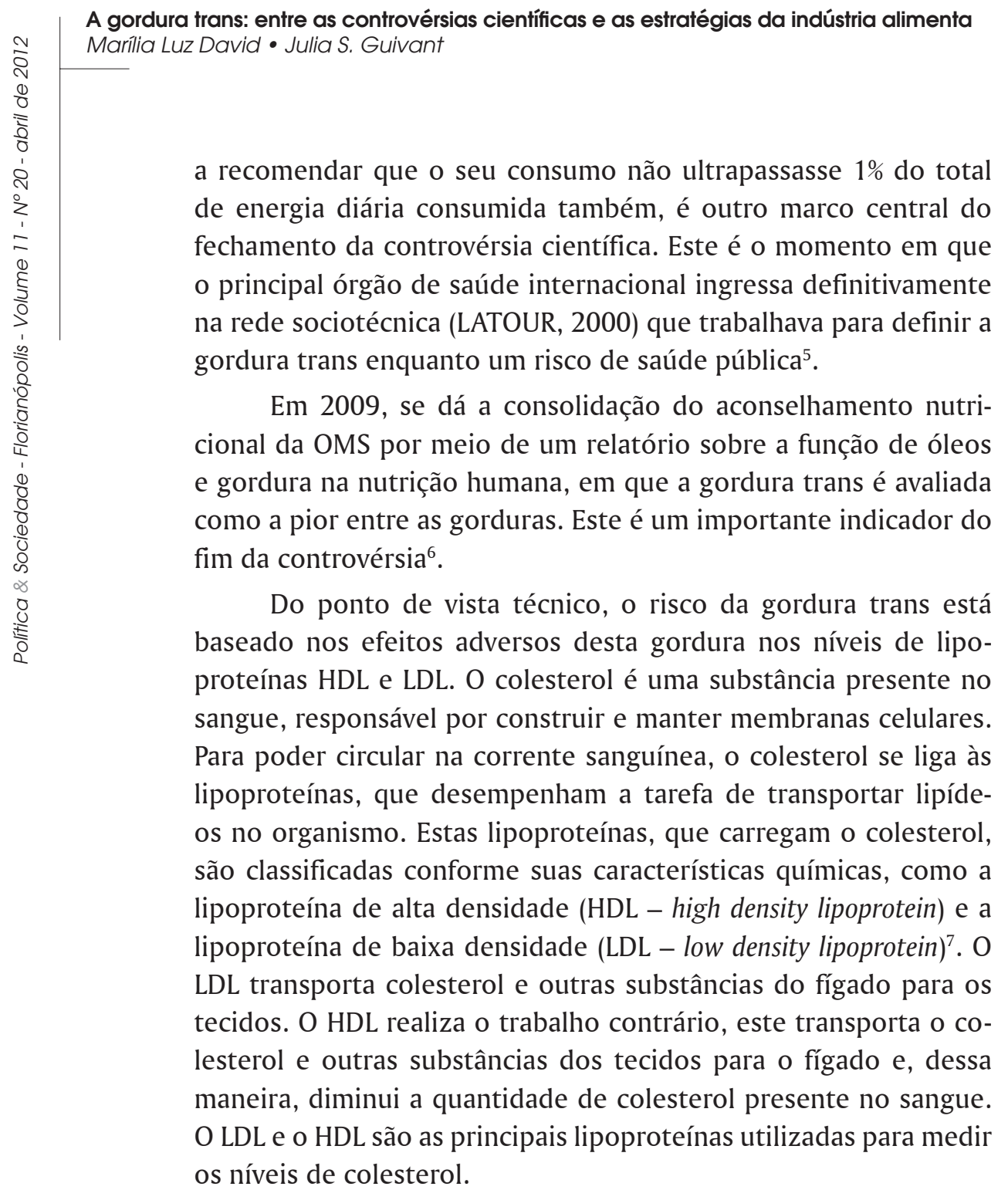

5 Seguimos um conceito mais geral de saúde pública que compreende dois aspectos: a) intervenções direcionadas a grupos ou populações e b) ações governamentais que visam à população, pois as intervenções propostas não podem ser atingidas pela iniciativa exclusiva dos indivíduos (DAWSON, 2007, p. 225).

6 Ainda assim isto não implica em um encerramento desta, pois apesar do risco ter se consolidado como uma caixa-preta, este pode voltar a ser questionado no futuro fazendo com que a controvérsia seja reaberta (LATOUR, 2000).

7 Existem outros tipos de lipoproteínas, mas para os fins deste trabalho apresentamos apenas estes dois tipos mais conhecidos. 
Durante o curso de vida ocorrem lesões nas paredes dos vasos sanguíneos provocadas por diversos fatores como o próprio envelhecimento do organismo, ou outras condições como a diabetes. O LDL é conhecido como "colesterol ruim", pois em altos níveis forma os chamados ateromas, isto é, placas que se depositam nestas lesões das paredes dos vasos sanguíneos e são formadas por substâncias liberadas pelo colesterol. Estas placas diminuem o diâmetro dos vasos sanguíneos e podem levar a sua obstrução. Por esse motivo, altos níveis de LDL colaboram para o desenvolvimento de doenças cardiovasculares. O HDL é conhecido como "colesterol bom", pois previne a formação destas placas que dificultam e podem cortar a irrigação sanguínea. Isto leva o HDL a ser considerado um fator de prevenção de doenças cardiovasculares. Os peritos responsáveis pelo relatório sobre a função de óleos e gordura na nutrição humana publicado pela OMS e FAO em 2009 consideraram que, a partir das pesquisas revisadas, a gordura trans tem efeitos particulares no colesterol, pois ao contrário de outros tipos de gorduras, ela não apenas reduz o colesterol LDL, mas também reduz o colesterol HDL (UAUY et al., 2009).

Durante a maior parte da controvérsia científica sobre a gordura trans, instituições de pesquisa financiadas pela indústria de alimentos e associações representantes desta refutaram as alegações de que a gordura trans teria efeitos adversos no colesterol e que, portanto, seria um fator na dieta que contribuía para a ocorrência de doenças cardiovasculares (KATAN; KORVER, 2006; DAVID, 2011). Além disso, a falta de iniciativas da indústria de reduzir o conteúdo de gordura trans durante a década de 1990 é um importante indicador desta postura ${ }^{8}$.

A indústria alimentar mudou sua postura frente ao risco e começou a reformular seus produtos para reduzir o conteúdo

8 A criação pela Unilever em 1994 da margarina Flora/Becel sem gordura trans abre uma exceção neste argumento. No entanto, esta iniciativa não puxou uma onda de reformulações por parte da indústria alimentar na época. Disponível em: $<$ http://www.florahearts.co.uk/Consumer/FooterArticle.aspx?Path=Common/Footer/ FooterArticles/AboutUs $>$. 
de gordura especialmente a partir de 2003 e 2004. Este período antecedeu o início das regras que tornaram obrigatória a rotulagem nutricional da gordura trans nos Estados Unidos e Canadá, e no qual a Dinamarca baniu a gordura trans dos alimentos comercializados no país. A pesquisadora norte-americana Nestle (2007) aponta que quando as regras de rotulagem entraram em vigor nos Estados Unidos já era muito difícil encontrar algum produto que não fosse rotulado como $0 \%$ gordura trans. Além disso, a indústria se tornou vulnerável a críticas uma vez que em 2003 a OMS revisou seu aconselhamento nutricional e passou a recomendar um consumo mínimo de gordura trans (OMS, 2003).

Esta onda de reformulações foi inaugurada pela Nestlé, que estendeu suas modificações para a redução dos níveis de sal em 2005, açúcar em 2007 e gordura saturada em 2009 (NESTLE, 2003). Em 2003 a Unilever comunicou que todas as suas linhas de margarinas seriam livres de gordura trans (FOOD NAVIGATOR, 2004). A Kraft Foods anunciou que retiraria a gordura trans de uma série de alimentos e em 2005 declarou que seu plano para reduzir o conteúdo de gordura trans em produtos se tornaria uma estratégia global (JUST-FOOD, 2005). Anteriormente, a Kraft tinha sido processada pelo fundador da campanha Ban Trans Fat por utilizar ingredientes com gordura trans em seus biscoitos Oreos, um dos principais produtos da empresa.

As ONGs de consumidores foram atores expressivos na controvérsia. As campanhas americans Trans Free America e a Ban Trans Fat (acima mencionada) da ONG de consumidores Center for Science in the Public Interest (CSPI) estão entre as que se destacaram por contribuir decisivamente para que grandes redes de restaurante fast food mudassem a composição de seus produtos com gordura trans. A primeira entre as grandes redes de restaurante fast food foi o Wendy's, após empresas como o McDonalds e o KFC se tornarem alvo de uma série de processos judiciais nos Estados Unidos (CSPI, 2007). O McDonalds foi obrigado a pagar sete milhões de dólares ao American Heart Association após não ter cumprido o anúncio feito em 2002 de que reduziria o conteúdo de gordura trans (NESTLE, 
2007, p. 387). Em 2008 a empresa divulgou que todas as batatas-fritas vendidas nos Estados Unidos e Canadá estavam livres de gordura trans e que em produtos disponíveis em países da Europa, América Latina, Ásia e Leste da África a quantidade fora reduzida para $0,5 \mathrm{~g} /$ porção (BLEIER, 2008).

Na Europa os supermercados parecem ter liderado esta tendência modificando produtos de suas próprias marcas: em 2005, uma das principais redes de supermercados do Reino Unido, a Marks \& Spencer's, anunciou que eliminaria a gordura trans de todos os seus produtos até o ano seguinte. Outras grandes redes supermercadistas inglesas, como a Sainsbury e Tesco, também anunciaram medidas parecidas (FOOD NAVIGATOR, 2005b). Em 2006, a British Retail Consortium, uma associação que representa os maiores supermercados do Reino Unido retirou a gordura trans de todos os alimentos de suas próprias marcas - decisão que afetou cerca de cinco mil produtos (BBC NEWS, 2007).

Outro momento desta mudança de posicionamento da indústria frente ao risco ocorre no Canadá em 2004. Após a introdução da rotulagem obrigatória da gordura trans em 2003, o governo canadense propôs medidas mais restritivas que reduziriam a presença desta gordura em alimentos no país. Em 2005, durante o período de consulta, foram ouvidos peritos da área da saúde e representantes de diversos setores da indústria de alimentos, como a Monsanto, Cargill, PepsiCo, o Canadian Council of Grocery Distributors, o Conseil de La Transformation Agroalimentaire et des Produits de Consommation, Dairy Farmers of Canada e o Canola Council of Canada. Durante a consulta, todas as empresas e associações representantes identificaram a gordura trans como um risco alimentar e concordaram com a ideia de que seria necessário reduzir o consumo de gordura trans no país (HEALTH CANADA, 2006).

Com estes exemplos voltamos ao argumento de que a indústria alimentar mudou sua postura e passou a reconhecer a gordura trans como um risco alimentar mais significativamente entre 20032004. Em sua análise a partir de relatórios de responsabilidade social-corporativa de empresas da indústria alimentar e de bebidas 
nos EUA e União Europeia, Herrick (2009) também identifica 2004 como um período em que o foco na saúde se torna parte-chave de estratégias da indústria alimentar para agregar valor a seus produtos.

No intuito de entender melhor esta inovação da indústria frente à consolidação do risco, no próximo item identificamos alguns aspectos sobre os riscos no sistema alimentar moderno e a dinâmica criada pela indústria com os alimentos sem gordura trans.

Quadro 1: Mudanças no perfil de consumo de gorduras no século XX

\begin{tabular}{|l|l|}
\hline Até 1950 & $\begin{array}{l}\text { Maior parte da gordura consumida é de origem } \\
\text { animal, proveniente da banha, leite carne e } \\
\text { manteiga. }\end{array}$ \\
\hline A partir de 1950 & $\begin{array}{l}\text { Consumo de óleos vegetais hidrogenados que } \\
\text { contêm gordura trans, mas com menor teor de } \\
\text { gordura saturada. } \\
\text { O aconselhamento nutricional de peritos da saúde } \\
\text { avaliou inicialmente a substituição de gorduras } \\
\text { animais por vegetais como mais saudável. } \\
\text { Incentivo também ao consumo de gordura } \\
\text { poliinsaturada como forma de prevenção a doenças } \\
\text { cardiovasculares. }\end{array}$ \\
\hline A partir de 1990 & $\begin{array}{l}\text { Questionamentos sobre os efeitos da gordura } \\
\text { trans na saúde e a utilização de óleos vegetais } \\
\text { hidrogenados. }\end{array}$ \\
\hline A partir de 2003-2004 & $\begin{array}{l}\text { Produtos sem gordura trans. } \\
\text { Crescem significativamente os investimentos em } \\
\text { pesquisa e produção de gorduras vegetais com } \\
\text { teor reduzido de gordura trans e gordura saturada. } \\
\text { Margarinas, por exemplo, incorporam uma série } \\
\text { de qualidades como vitaminas A, D e E, gorduras } \\
\text { poliinsaturadas, redução das quantidades de gordura } \\
\text { saturada e eliminaça da gordura trans. } \\
\text { Variações nas regulações nacionais na definição de } \\
\text { "0\% gordura trans". }\end{array}$ \\
\hline
\end{tabular}




\section{Os riscos da gordura trans}

Parte da consolidação da gordura trans como risco ocorreu, entre outros motivos, devido à maneira de avaliar o alimento baseada no nutriente. Historicamente, o nutricionismo indica o sucesso de uma maneira particular da ciência da Nutrição mobilizar alimentos para dentro de sua rede (LATOUR, 2000) e produzir conhecimento. Além disso, a consolidação do risco da gordura trans também está baseada na progressiva especialização do conhecimento característico da modernidade (GIDDENS, 2002, p. 35). O risco alimentar da gordura trans, como parte do nutricionismo, é um indicador no sistema alimentar deste traço institucional moderno mais amplo relacionado à especialização do conhecimento.

A utilização de alegações de saúde se tornou uma aposta bem-sucedida para a indústria: nos Estados Unidos, os alimentos que traziam a afirmação "livre de gordura trans" tiveram um crescimento de $12 \%$ em suas vendas em 2004 (FOOD NAVIGATOR, 2005a). No Brasil, enquanto que em 2007 apenas 10\% dos alimentos traziam este tipo de informação, este número saltou para 64 em 2008 (BOTELHO, 2008). Ao mesmo tempo em que a retirada da gordura trans se tornou uma nova qualidade (CALLON; MÉADEL; RABEHARIOSA, 2002) que expande o leque de atributos que tornam o alimento mais saudável, a quase onipresença das alegações de saúde " $0 \%$ gordura trans" em rótulos de alimentos processados indica que a indústria acabou encurralada e se viu forçada a reconhecer a gordura trans como risco.

Diante das críticas que responsabilizam a indústria alimentar em parte pelo aumento de doenças crônicas (OMS, 2002a; OMS, 2004), a criação de produtos com diferentes benefícios nutricionais é uma das principais estratégias utilizadas pelas empresas para sair da posição de culpadas. Este é um aspecto importante dos alimentos sem gordura trans, pois a consolidação do risco desta gordura enquanto um risco de saúde pública era potencialmente problemática para a indústria alimentar caso esta continuasse na posição de discordante, conforme o fez durante a maior 
parte da controvérsia científica (DAVID, 2011). O reconhecimento da gordura trans como risco alimentar pela OMS, por exemplo, foi decisivo para a expansão do reconhecimento da gordura trans como risco para outros países. Até 2006, em países como o Chile, Argentina, Paraguai, Uruguai, Rep. Dominicana, Costa Rica e Brasil já existiam propostas para a rotulagem da gordura trans que tomavam o aconselhamento nutricional da OMS como justificativa. A Organização Pan-Americana de Saúde (OPAS) divulgou em 2008 a Declaração do Rio: "Américas livres de gordura trans", que utiliza a Global Strategyon Diet, Physical Activity and Health da OMS como marco. Entre os principais objetivos elencados pelo documento está a substituição da gordura trans e sua rotulagem obrigatória nos alimentos processados (OPAS/ OMS, 2008, p. 4-5). A indústria alimentar se viu forçada a reconhecer a gordura trans como risco; no entanto, contra as expectativas de autoridades nacionais, ONGs de consumidores e órgãos internacionais de saúde que reivindicavam a existência de um risco de saúde pública, a indústria amarrou o risco da gordura trans a outros elementos.

Os produtos sem gordura trans engendram uma versão de risco em que a indústria também é um elemento indispensável na resolução do problema do aumento de doenças relacionadas à alimentação. A questão seria que, quando começa a produzir os alimentos sem gordura trans, e toma o discurso da saúde para si, a indústria procura se tornar a solução de um problema que formula em seus próprios termos.

A indústria necessitava de uma inovação que fosse lucrativa, mas que também anunciasse estas reformulações, para que deixasse de ser vulnerável a possíveis críticas em relação a este risco em particular. Só restava à indústria tentar reverter a situação. Se durante a década de 1990 as propostas para remover a gordura trans dos alimentos foram recebidas com resistência por grande parte da indústria, a partir de 2003-2004 estas passaram a ser vistas como uma nova maneira de rebater críticas e trazer a preocupação com a saúde para o mercado global de alimentos. Iniciativas de grandes empresas sinalizam este movimento de preocupação com 
a saúde, como a Unilever que revisou o seu portfólio de alimentos e bebidas para aumentar o número de opções de alimentos com um perfil mais saudável em 2004 (PATTON, 2005) e a Kraft Foods, que criou em 2005 o selo Sensible Solutions para identificar aqueles alimentos que seguem critérios nutricionais estabelecidos pelas Diretrizes Alimentares Americanas (FOOD NAVIGATOR, 2007).

\section{Considerações finais}

Um aspecto importante da produção de conhecimento no campo da Nutrição diz respeito à valorização do nutriente como medida-chave para avaliar a qualidade do alimento (SCRINIS, 2008). O nutriente é fundamental para o aconselhamento nutricional que os peritos promovem e, portanto, para a avaliação do que seria um risco alimentar. A retirada da gordura trans como uma nova maneira de qualificar o alimento está relacionada a esta decomposição deste em conjuntos de nutrientes.

O reconhecimento de lacunas na perícia e da existência de riscos alimentares pelos consumidores são dois traços da vivência dos riscos (GIDDENS, 1991) discutidos aqui. Ainda no início da controvérsia científica em torno da gordura trans, autoridades nacionais americanas reconheceram a dificuldade em explicar para o público que o óleo vegetal hidrogenado (com gordura trans), um ingrediente até então considerado mais saudável do que a gordura animal, poderia provocar danos à saúde. Em 1992, um especialista em óleos comestíveis do Departamento de Agricultura americano comentou durante entrevista ao jornal americano The New York Times: "“It's a nightmare. (...) It's really a nasty thing when you try to explain it. There's total confusion for consumers" (BURROS, 1992). Esta avaliação nutricional que, em retrospectiva, se tornou um engano, ilustra bem estes traços da vivência dos riscos que colaboram para que os paradoxos da alimentação, especialmente aquele entre saúde e doença, se tornem mais aparentes. Estes paradoxos alimentares ainda convivem com um modelo que dá valor ao corpo esguio 


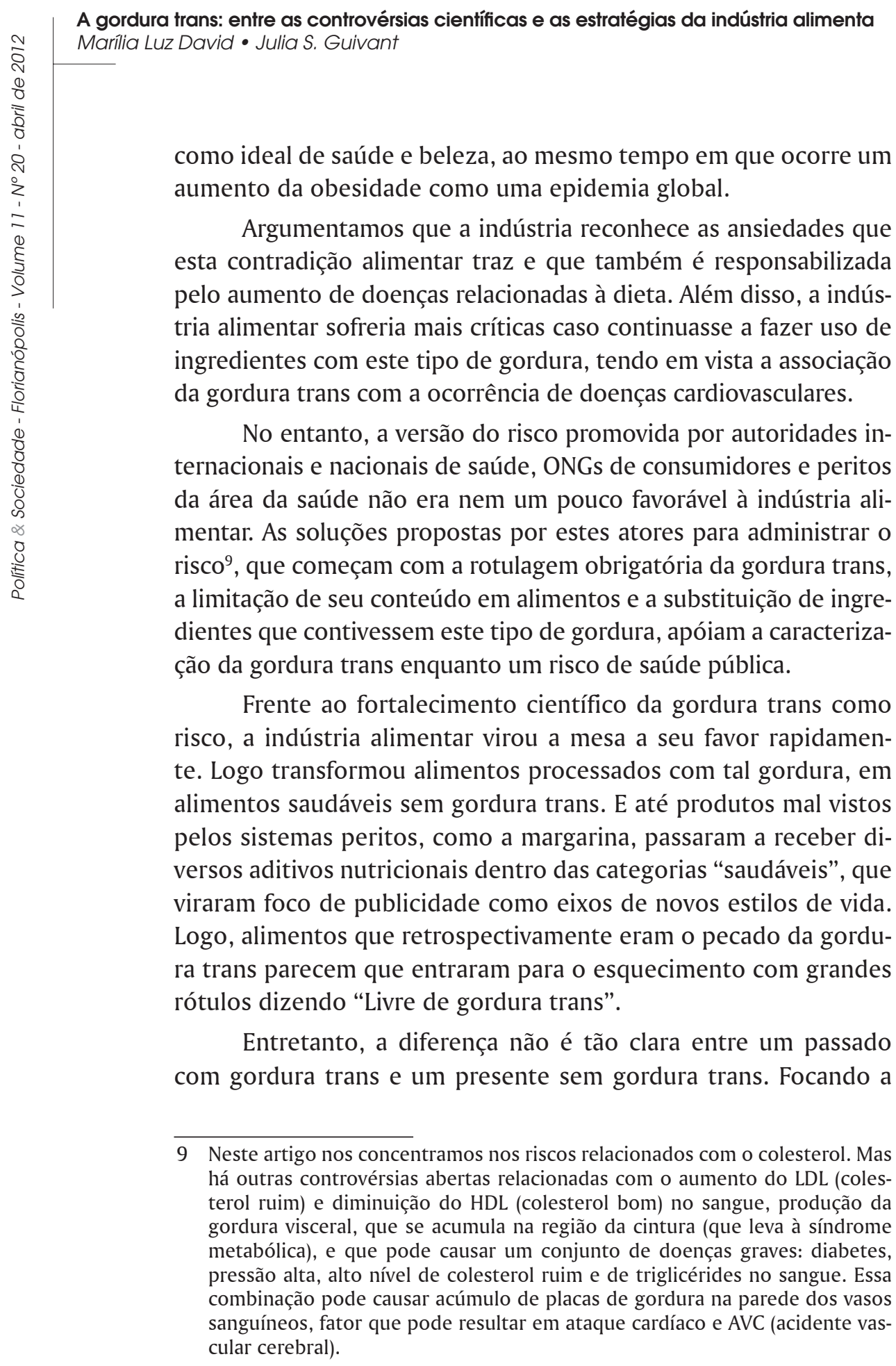


situação no Brasil, podemos destacar a determinação da Agência Nacional de Vigilância Sanitária (Anvisa) em 2006 que estabeleceu que o item "gordura trans" passasse a ser impresso na tabela nutricional do rótulo dos alimentos. Com isso, as empresas foram obrigadas a especificar, além do teor de lipídeos e de gorduras saturadas, a quantidade total de ácidos graxos trans presentes nos produtos.

Mas, o que aparece ainda invisível ao consumidor que procura saúde é que as regras de rotulagem nutricional, como as existentes no Brasil, permitem que o alimento contenha até $0,2 \mathrm{~g}$ por porção de gordura trans para ser classificado como "livre de gordura trans". $\mathrm{O}$ mesmo ocorre em outros países que estabeleceram regras para a rotulagem da gordura trans, como os EUA, a Dinamarca e o Canadá - ainda que existam variações nos limites estabelecidos para que um alimento seja considerado isento de gordura trans.

Ainda que em quantidades bem menores, a gordura trans fabricada industrialmente continua presente em alimentos processados. Dessa maneira, uma pessoa que se alimenta diariamente com diversos produtos " $0 \%$ gordura trans" não está totalmente livre desta gordura em seu cotidiano. A OMS recomenda que a ingestão de gordura trans não ultrapasse $1 \%$ do valor calórico da dieta, o que numa alimentação de 2.000 calorias equivale a dois gramas diários de gordura trans - quantidade equivalente a três biscoitos recheados de morango. De acordo com a Associação Brasileira das Indústrias da Alimentação (Abia), apenas 30\% dos biscoitos vendidos no Brasil são livres de gordura trans (VEJA, 2008 ${ }^{10}$ ). Mas, por exemplo, se metade de uma bolacha tem a quantia total de $0,2 \mathrm{~g}$ de gordura trans, quem come meio biscoito calculando a quantidade de gordura trans consumida? Nem pensar o que se consome nas situações de comer fora da casa, e não só em restaurantes fast foods.

Justamente por colocar as alegações de saúde nos rótulos e nas campanhas publicitárias, a Abia conseguiu, até o momento de redação deste artigo (fevereiro de 2012), bloquear judicialmente a

10 Disponível em: < http://veja.abril.com.br/idade/exclusivo/perguntas_respostas/gordura-trans/index.shtml > . Consultado em: 26/02/2012. 
vigência da resolução da diretoria colegiada (RDC) no 24/2010, da ANVISA, que normatizava a propaganda de alimentos com elevados teores de açúcar, gordura trans, sódio e de bebidas com baixo valor nutricional. Em um documento ${ }^{11}$ produzido pela Abia para esclarecer sobre a gordura trans em alimentos e seus efeitos na saúde, a associação assume que ainda não há como substituir completamente seu uso e recomenda aos consumidores que, caso tenham alguma dúvida sobre os riscos, procurem "instituições científicas" para se informar. Entretanto, o que o consumidor médio brasileiro entende por "instituições científicas" quando não se da nem sites ou telefones de consulta? Apesar das evidências dos riscos à saúde da gordura trans, esta continua presente, mas oculta para os consumidores atrás de rótulos que mostram o contrário, enquanto, pelo menos a ABIA no Brasil, se mostra resistente a informar melhor aos consumidores a respeito dos produtos que consomem.

Original recebido em: 07/12/2011 Versão final recebida em: 16/03/2012

\section{Referências}

ANDERSON, J. T.; GRANDE, F.; KEYS, A. Hydrogenated Fats in the Diet and Lipids in the Serum of Man.The Journal of Nutrition, $v$. 75, p. 388-394, 1961.

BBC NEWS. Retailers to stop trans-fat use. BBC News, 31 janeiro 2007. Disponível em: <http://news.bbc.co.uk/2/hi/health/6314753.stm>.

BEARDSWORTH, A.; KEIL, T. Sociology on the Menu: an invitation to the study of food and society. Londres: Routledge, 1997.

BLEIER, K. McDonald's fries are now trans fat-free in U.S., Canada. USA Today, 22 maio 2008. Disponível em: < http://www.usatoday.

11 Disponível em: < http://www.abia.org.br/anexos/07QA-ABIAsobregordurastrans-FINAL. pdf. Consultado em: 26/02/ 2012>. 
com/money/industries/food/2008-05-22-mcdonaldstrans-fat_N. htm>.

BOTELHO, R. Cai quantidade de gordura trans em panetones. Folha de São Paulo, São Paulo, 20 dezembro 2008. Disponível em: $<$ http://www1.folha.uol.com.br/fsp/saude/sd2012200803.htm>.

BURRCH, D.; LAWRENCE, G. The "Wellness" Phenomenon: Implications for Global Agri-food Systems. In: LAWRENCE, G.; LYONS, K.; WALLINGTON, T. (Org.). Food Security Nutrition and Sustainability. Londres: Earthscan, 2010.

BURROS, M. Now What? U.S. Study Says Margarine May Be Harmful. The New York Times, Nova York, 7 outubro 1992. Disponível em: $<$ http://www.nytimes.com/1992/10/07/garden/now-what-us-studysaysmargarine-may-be-harmful.html?scp $=9 \& \mathrm{sq}=$ trans + fat $\& s t=$ nyt $>$.

BURROS, Marian. Eating Well. The New York Times, Nova York, 2 março 1994. Disponível em: < http://www.nytimes.com/1994/03/02/ garden/eating-well.html?scp $=12 \& \mathrm{sq}=$ trans + fat $\& s t=$ nyt $>$.

CALLON, M.; MÉADEL, C.; RABEHARISOA, V. The economy of qualities. Economy and Society, v. 31, n. 2, p. 194-217, 2002.

CSPI. Burger King Hit With Trans Fat Lawsuit. Center For Science in the Public Interest, 16 maio 2007. Disponível em: <http://www. cspinet.org/new/200705161.html>.

DAVID, M. L. 0\% gordura trans: uma análise da construção de riscos alimentares. Florianópolis. Dissertação (Mestrado em Sociologia Política). Universidade Federal de Santa Catarina, 2011.

DAWNSON, A. Food and the Public's Health. Journal of Agricultural and Environmental Ethics, v. 20, n. 3, p. 225-229, 2007.

FOOD NAVIGATOR. Unilever Canada takes the 'trans' out of fat. Food Navigator,24março2004.Disponívelem: $<$ http://www.foodnavigator. com/Financial-Industry/Unilever-takes-the-transout-of-fat $>$. 
A gordura trans: entre as controvérsias científicas e as estratégias da indústria alimenta Marilia Luz David • Julia S. Guivant

FOOD NAVIGATOR. Trans fat label law offers threats and opportunities. Food Navigator - USA, 1 julho 2005a. Disponível em: $\quad<$ http://www.foodnavigator-usa.com/Legislation/Trans-fatlabel -law-offersthreats-and-opportunities $>$.

FOOD NAVIGATOR. Marks\& Spencer bans hydrogenated fats. Food Navigator, 24 novembro 2005b. Disponível em: < http://www. foodnavigator.com/Science-Nutrition/Marks-Spencer-banshyd rogenated-fats $>$.

FOOD NAVIGATOR. Kraft reveals its four approaches to health and wellness. Food Navigator-USA, 29 agosto 2007. Disponível em: <http://www.foodnavigator-usa.com/Business/ Kraft-reveals-its-four-approaches-to-health-and-wellness $>$.

GIDDENS, Anthony. As consequências da modernidade. São Paulo: Editora Unesp, 1991.

GIDDENS, A. Modernidade e identidade. Rio de Janeiro: Jorge Zahar, 2002.

GUIVANT, J. Reflexividade na sociedade de risco: conflitos entre leigos e peritos sobre agrotóxicos. In: HERCULANO, S. (Org.). Qualidade de vida e riscos ambientais. Niterói: Ed. da UFF, 2000.

GUIVANT, J. Global food risks: environmental and health concerns in Brazil. In: HOGAN, D. J.; TOLMASQUIM, M. T. (eds). Human dimensions of global environmental change. Rio de Janeiro: Academia Brasileira de Ciências, 2001.

HEALTH CANADA. TRANSforming the food supply - Report of the Trans Fat Task Force Submitted to the Minister of Health. Ottawa: junho de 2006. Disponível em: <www.healthcanada.ca/transfat>.

HERRICK, C. Shifting blame/ selling health: corporate social responsibility in the age of obesity. Sociology of Health \& Illness, v. 31, n. 1, p. 51-65, 2009. Disponível em: < http://www3.interscience. wiley.com/cgi-bin/fulltext/121391015/PDFSTART > . 
HU, F. B., et al. Dietary Fat Intake and the Risk of Coronary Heart Disease in Women. The New England Journal of Medicine, Waltham, v. 337, n. 21, 1997. Disponível em: < http://content.nejm. org/cgi/content/full/337/21/1491>.

INTERNATIONAL FOOD AND INFORMATION COUNCIL. 2008 Food \& Health Survey: Consumer Attitudes toward Food, Nutrition \& Health. Washington: International Food and Information Council, 2008.

KATAN, M. B.; KORVER, O. The Elimination of Trans Fats from Spreads: How Science Helped to Turn an Industry Around. Nutrition Reviews, v. 64, n. 6, p. 275-279, 2006.

JUDD, J. T. et al. Dietary trans fatty acids: effects of plasma lipids and lipoproteins on healthy men and women. American Journal of Clinical Nutrition, v. 59, n. 4, 1994. pp. 861-868.

JUST-FOOD. U.S.: Kraft completes trans fat reformulation. JustFood, 21 dezembro 2005. Disponível em: <http://www.just-food. $\mathrm{com} /$ news/kraft-completes-trans-fat-reformulation_id86100. aspx $>$.

LANG, T.; HEASMAN, M. Food Wars: The Global Battle for Mouths, Minds and Markets. Londres: Earthscan, 2004.

LATOUR, B. Ciência em ação: como seguir cientistas e engenheiros sociedade afora. São Paulo: Ed. UNESP, 2000.

MATTSON, F. H.; HOLLENBACH, E. J.; KLIGMAN, A. M. Effect of hydrogenated fat on the plasma cholesterol and triglyceride levels of man. American Journal of Clinical Nutrition, v. 28, n. 7, p. 726$731,1975$.

MENSINK, R. P.; KATAN, M. B. Effect of Dietary Trans-Fatty Acids on Hight Density and Low-Density Lipoprotein Cholesterol Level in Healthy Subjects. The New England Journal of Medicine, v. 323, n. 7, 1990. pp. 439-445. 
A gordura trans: entre as controvérsias científicas e as estratégias da indústria alimenta Marilia Luz David • Julia S. Guivant

MOZZAFFARIAN, D.; ARO, A.; WILLETT, W. C. Health effects of transfatty acids: experimental and observational evidence. European Journal of Clinical Nutrition, v. 63, 2009. pp. 5-21 (suplemento).

NESTLE. Delivering against our nutrition policies, 2003. Disponível em: <http://www.nestle.com/CSV/Nutrition/Making NutritionThePreferredChoice/Pages/DeliveringAgainstPolicies. aspx $>$.

NESTLE, M. Food politics: how the food industry influences nutrition and health. Berkeley/ Los Angeles: University of California Press, 2007.

OMS/FAO. Fats and Oils in human nutrition. Report of a Joint FAO/ WHO Expert Consultation. Roma: OMS/FAO, 1994.

OMS. Globalization, Diets and Noncommunicable Diseases. Genebra: OMS, 2002a.

OMS. World Health Report 2002: Reducing Risks, Promoting Healthy Life. Genebra: OMS, 2002b.

OMS. Diet, Nutrition and prevention of chronic diseases. Genebra: OMS/FAO, 2003. (WHO Technical Report Series 916).

OMS. Global Strategy on Diet, Physical Activity and Health Report of a Joint WHO/FAO Expert Consultation. Genebra: OMS, 2004.

OOSTERVEER, P.; GUIVANT, J. S.; SPAARGAREN, G. Alimentos verdes em supermercados globalizados: uma agenda teóricometodológica. In: GUIVANT, J. S.; SPAARGAREN, G.; RIAL, C. (Org). Novas práticas alimentares no mercado global. Florianópolis: Ed. da UFSC, 2010.

OPAS/OMS. As Américas Livres de Gordura Trans: Declaração do Rio de Janeiro. In: Reunião Interamericana, em nível ministerial, sobre saúde e agricultura (RIMSA), 15ạ, 2008, Rio de Janeiro. Agricultura e saúde: aliança pela igualdade e desenvolvimento rural nas Américas. Washington: OPAS/OMS, 2008. 
PATTON, D. Healthy innovation attractive to investors. Beverage Daily, 9 setembro, 2005. Disponível em: < http://www.beveragedaily. com/Financial/Healthy-innovation-attractive-to-investors $>$.

POLLAN, M. Em defesa da comida. Rio de Janeiro: Intrínseca, 2008. POPKIN, B. M. Technology, transport, globalization and the nutrition transition food policy. Food Policy, v. 31, n. 6, p.554569, 2006. Disponível em: <http://www.sciencedirect.com/ science?_ob $=$ PublicationURL\&_cdi $=5950 \&$ _pubType $=J \&$ acct $=$ C000050221\&_version $=1 \&$ urlVersion $=0$ \&_userid $=10 \& \mathrm{~m}$ d5=3d64916799f80f5e412a970806266c7a\&jchunk=31\#31>.

ROZIN, P. Food is Fundamental, fun, frightening, and far-reaching. Social Research, v. 66, n. 1, p. 9-30, 1999.

SENTI F. R. (ed.). Health aspects of dietary trans fatty acids. Bethesda: Life Sciences Research Office, Federation of American Societies for Experimental Biology, 1985.

SCARBROUGH, F. E. Some Food and Drug Administration perspectives of fat and fatty acids. American Journal of Clinical Nutrition, v. 65, n. 5, 1997. pp. 1578-1580 (suplemento).

SCRINIS, Gyorgy. On the Ideology of Nutritionism. Gastronomica: The Journal of Food and Culture, v. 8, n. 1, 2008. Disponível em: $<$ http://www.gyorgyscrinis.com $>$.

STENDER, S. et al. The influence of trans fatty acids on health: a report from The Danish Nutrition Council. Clinical Science, v. 88, n. 4 , 1995. pp. 375-392.

UAUY et al. WHO Scientific Update on trans fatty acids: summary and conclusions. European Journal of Clinical Nutrition, v. 63, 2009. pp. 68-75 (suplemento)

VEJA. Gordura Trans, Seções On-Line: Perguntas\&Respostas. Revista VEJA, setembro, 2008. Disponível em: <http://veja.abril.com.br/ idade/exclusivo/perguntas_respostas/gordura-trans/index.shtml $>$. 
VERGROESEN, A. J. Dietary fat and cardiovascular disease: possible modes of action of linoleic acid. Proceedings of the nutrition society, v. 31, p. 323-329, 1972.

VIALTA, A. et al. Brasil food trens 2020. São Paulo: Ideal, 2010.

WILLETT, W. C. et al. Intake of trans fatty acids and risk of coronary heart disease among women. Lancet, v. 341, 1993. pp. 581-585.

WILLETT, W. C.; ASCHERIO, A. Trans Fatty Acids: Are the Effects Only Marginal? American Journal of Public Health, v. 84, n. 5, 1994. pp. 722-724.

\begin{abstract}
Trans fat: between scientific controversies and strategies of the food industry

Among modern food risks, trans fat stands out as one of which has become part of everyday consumer most recently. Industrially manufactured trans fat was initially considered a healthier alternative to food production and it became widely used by the food industry since the second half of the twentieth century. This fat was considered a healthier substitute to animal fat. However, a scientific controversy started in 1990 due to the publication of an article in a leading medical journal which related the consumption of trans fat to cardiovascular disease. In this article first we analyze how trans fat became a dietary risk and secondly what were the changes made by the food industry. Finally we point out the paradox that although "trans fat free" labels can be found everywhere, it remains present in everyday life, consumed in small quantities in different products.
\end{abstract}

Keywords: food-risks, labeling, risk regulation, health food. 